\title{
A Patient Presenting with Severe Hypoglycaemia and Autonomic Instability Found to Have Multiple Myeloma Complicated with Amyloidosis
}

Kushalee P Jayawickreme, Shyama Subasinghe, Rochana De Silva, Preethi Dissanayake, Lasanthi Perera

Sri Jayawardenepura General Hospital, Sri Jayawardenepura Kotte, Sri Lanka

$\mathrm{B}$ ackground: Multiple myeloma and its potential complication, amyloidosis, both have multisystem involvement. Immunoglobulin light chain (AL) amyloidosis is rare, affecting an estimated 5-12 people per million, per year. However, only $10-15 \%$ of amyloidosis cases are associated with multiple myeloma, and 30\% of multiple myeloma cases can be complicated by amyloidosis. Case presentation: A 72-year-old female presented with generalised body weakness, significant loss of appetite and loss of weight for six months. She had backache for two months and bilateral lower limb burning pain and numbness. She had spinal tenderness over the first and second lumbar vertebrae (L1, L2), bilateral lower limb glove and stocking numbness, and hepatomegaly. X-rays showed wedge fractures at the L1 and L2 vertebral level. Haematological analysis showed normochromic normocytic anaemia with moderate rouleaux formation. Serum protein electrophoresis was consistent with the diagnosis of multiple myeloma. The patient was readmitted a few days later with transient slurring of speech, lightheadedness and urinary retention. She had a blood sugar level of $39 \mathrm{mg} / \mathrm{dL}$, without hypoglycaemic awareness, and had a low blood pressure reading of 70/40 $\mathrm{mmHg}$ with postural drop. She had recurrent episodes of both hypoglycaemia and hypotension. An ultrasound of the patient's abdomen revealed hepatomegaly with normal sized kidneys. She had significant proteinuria with upper normal renal functions. The liver biopsy showed apple green birefringence on congo red staining and AL amyloidosis was confirmed. Conclusion: This patient has multi-organ involvement leading to autonomic instability and hypoglycaemia, as a result of multiple myeloma and the co-existence of amyloidosis, which is a known complication of myeloma.

\section{Keywords}

Multiple myeloma, immunoglobulin light chain amyloidosis, hypoglycaemia, autonomic neuropathy

Disclosures: Kushalee P Jayawickreme, Shyama Subasinghe, Rochana De Silva, Preethi Dissanayake and Lasanthi Perera have no conflicts of interest to declare in relation to this article.

Acknowledgements: The authors express their gratitude to the patient who kindly gave consent for this case to be presented in this paper.

Review Process: Double-blind peer review.

Compliance with Ethics: All procedures were followed in accordance with the responsible committee on human experimentation and with the Helsinki Declaration of 1975 and subsequent revisions, and informed consent was received from the patient involved in this case study.

Authorship: All named authors meet the criteria of the International Committee of Medical Journal Editors for authorship for this manuscript, take responsibility for the integrity of the work as a whole and have given final approval for the version to be published.

Received: 25 September 2019

Accepted: 18 November 2019

Citation: European Oncology \& Haematology, 2019;15(2):106-8

Corresponding Author: Kushalee P Jayawickreme, Sri Jayawardenepura General Hospital, Sri Jayawardenepura Kotte, Sri Lanka. E: kushaleej5@gmail.com

Support: No funding was received in

the publication of this article.
Amyloidosis is a group of diseases resulting from intracellular and extracellular deposition of insoluble abnormal amyloid fibrils, which alters the normal function of tissues. Glycosaminoglycans, apolipoprotein-E and serum amyloid P component comprise 10\% of deposits, while amyloid fibrils formed by misfolded proteins comprise $90 \%$ of deposits. Amyloidosis can locally target a single organ or can have multisystem involvement. ${ }^{1}$

Amyloidosis is classified by the biochemical structure of the amyloid fibrils. Amyloid type A amyloidosis is secondary to chronic inflammatory disease. AL amyloidosis is either primary or is associated with lower grade plasma cell clones, but it can occur with multiple myeloma or, less often, with Waldenström's macroglobulinaemia or non Hodgkin's Iymphoma. ${ }^{2}$ AL Amyloidosis is rare, affecting an estimated 5-12 people per million per year. ${ }^{3}$ However, only $10-15 \%$ of amyloidosis cases are associated with multiple myeloma. ${ }^{4}$ Overall, $30 \%$ of multiple myeloma cases can be complicated by primary subclinical amyloidosis. ${ }^{5}$

\section{Case Presentation}

A 72-year-old female with diabetes mellitus for two years presented with generalised body weakness, significant loss of appetite and loss of weight over the past six months. She had backache for two months and bilateral lower limb burning pain and numbness. On examination she was haemodynamically stable. She had spinal tenderness over the first and second lumbar vertebrae (L1, L2), lower limb muscle wasting and glove and stocking bilateral lower limb sensory impairment. She had a slightly large tongue. Hepatomegaly was noted, with no splenomegaly. Haemoglobin ( $\mathrm{Hb}$ ) was $10.5 \mathrm{~g} / \mathrm{dL}$, platelet count was 386 , erythrocyte sedimentation rate (ESR) was 60, alkaline phosphatase was $470 \mathrm{IU} / \mathrm{L}$. X-rays showed wedge fractures at L1 and L2 vertebrae, but no lytic lesions were picked up in the rest of the skeletal survey. Haematological analysis showed normochromic normocytic anaemia with moderate rouleaux formation, mild polymorphonuclear leucocytosis, occasional hypersegmented neutrophils, and plasmacytoid lymphocytes. Urine was negative for Bence-Jones proteins, but serum protein electrophoresis had a monoclonal band in the gamma region. Serum erythropoietin levels were at the upper limit of normal at $31.4 \mathrm{mU} / \mathrm{mL}$. Bone marrow aspiration biopsy showed $15 \%$ of the nucleated marrow cells to be an abnormal population of plasma cells with blasts $<3 \%$, indicating clonal plasma cell 
proliferation consistent with the diagnosis of multiple myeloma. Flow cytometry was positive for CD38, CD138, CD56, CD38/intra-cytoplasmic immunoglobulin (cy-lg) lambda and negative for cy-lg kappa. The patient was started on analgesics, vitamin D and zolendronate. The wedge fracture was managed conservatively and she was referred to an oncologist for chemotherapy.

One week after initial presentation and prior to her intended chemotherapy commencement date, the patient was readmitted with transient slurring of speech and lightheadedness. Her blood sugar level was $39 \mathrm{mg} / \mathrm{dL}$, without hypoglycaemic awareness, and she had a low blood pressure reading of $70 / 40 \mathrm{mmHg}$, with postural drop. She had recurrent episodes of hypotension, which required fluid resuscitation and ionotropes, and urinary retention suggestive of autonomic instability. An electrocardiogram (ECG) showed no ischaemic changes or arrhythmia, a two-dimensional echocardiogram was normal, and magnetic resonance imaging of the brain was normal. She was treated for possible neuroglycopenia causing a transient ischaemic attack. Her oral hypoglycaemic treatments sitagliptin (50 mg twice-daily) and metformin (500 mg twice-daily) were withheld, and 50\% dextrose was given. She had recurrent episodes of severe hypoglycaemia despite withholding her oral hypoglycaemic medication and subsequent correction with dextrose. Her HDA1C value was 6.5\%, and fasting blood sugar was low at $67 \mathrm{mg} / \mathrm{dL}$. Since her diagnosis of diabetes mellitus, which was two years previous, the patient has maintained good glycaemic control. She also persistently had normal serum creatinine levels during routine check-up, thus had not had her urine protein creatinine ratio checked until now.

An ultrasound of the patient's abdomen showed hepatomegaly with heterogeneous echotexture and reduced parenchymal attenuation, with normal sized kidneys, and liver enzymes were normal. Serum creatinine was $110 \mu \mathrm{mol} / \mathrm{L}$, estimated glomerular filtration rate was 56 . Her urine protein creatinine ratio was 2526 . However, there was notable absence of diabetic retinopathy. Serum albumin was low at $2.6 \mathrm{~g} / \mathrm{L}$, with an albumin to globulin ratio of 1 . This required intravenous albumin replacement. Calcium ions $\left(\mathrm{Ca}^{2+}\right)$ were high at $10.2 \mathrm{mg} / \mathrm{dL}$, sodium ions $\left(\mathrm{Na}^{+}\right)$were slightly low at $130 \mathrm{mmol} / \mathrm{L}$, and potassium was normal at $4.3 \mathrm{mmol} / \mathrm{L}$. Normal early morning cortisol levels were noted.

A contrast-enhanced computed tomography scan of the abdomen showed no evidence of any other organ malignancy or lymphadenopathy, and tumour markers were normal. Liver biopsy showed apple green birefringence on Congo red staining and AL amyloidosis was confirmed. Rectal biopsy showed normal rectal mucosa but the patient developed uncontrollable rectal bleeding following the biopsy, despite a normal clotting profile. The bleeding was controlled by tight packing and required blood transfusion.

The patient refused renal biopsy to confirm renal amyloidosis as the cause for significant proteinuria and to exclude diabetic nephropathy. A lower limb nerve conduction study showed sensory and motor axonal peripheral neuropathy. A sural nerve biopsy was offered to the patient in order to confirm amyloid neuropathy, but she refused this procedure. Following referral to an oncologist, vertebral radiotherapy was given for backache, which was likely due to myelomatous involvement of the bone. Chemotherapy was started with bortezomib, melphalan and prednisolone.

\section{Discussion}

Amyloidosis, as well as multiple myeloma, can have multi-organ involvement. It is likely that AL amyloidosis is the most common cause of acquired amyloid peripheral neuropathy, as peripheral neuropathy is seen in $17 \%$ cases of AL amyloidosis. ${ }^{6}$ Sensory motor axonal polyneuropathy, as observed in this patient, and carpal tunnel syndrome are the neuropathy types most frequently associated with AL amyloidosis. There are also reported cases of mononeuropathies, mononeuritis multiplex, lumbosacral radiculoplexopathy and chronic inflammatory demyelinating polyneuropathy. Symptoms typically begin with painful paraesthaesia of legs, as in this case. AL Amyloid autonomic neuropathy is found in $65 \%$ of patients with amyloid peripheral neuropathy. ${ }^{6}$ Our patient had features of autonomic neuropathy such as recurrent hypotensive episodes, hypoglycaemic unawareness, urinary retention and uncontrolled rectal bleeding, which is also due to impaired vasoconstrictor response of vasculature to trauma. Diabetes mellitus alone can also lead to a similar presentation. Patients who had been diagnosed with diabetes for more than three years were found to be 7.8 times more likely to develop diabetic neuropathy, and patients with diabetes who had a HbA1c level of more than $6.5 \%$ were found to be 16.9 times more likely to develop diabetic neuropathy? In the case presented here, the patient's duration of diabetes was only two years, and the condition was persistently well controlled with a HbA1c of $6.5 \%$. This suggests that amyloidosis was a more likely cause for this patient's neuropathy.

It has been shown that $10 \%$ of acquired polyneuropathies in adults to be associated with a monoclonal gammopathy and, of those, the most common is IgM monoclonal gammopathy which is associated with a $50 \%$ incidence of polyneuropathy. ${ }^{8}$ More than $50 \%$ of IgM paraproteins react with myelin associated glycoprotein (MAG) in the central and peripheral nervous system resulting in 'anti-MAG paraproteinemic demyelinating peripheral neuropathies' ${ }^{8}$ Multiple myeloma alone can also cause peripheral neuropathy due to perineural or perivascular deposition of paraproteins. Alternatively, paraproteinaemias or plasma cell dyscrasias can be linked to POEMS (Polyneuropathy, Organomegally, Endocrinopathy, Monoclonal protein, Skin changes) syndrome. The diagnostic criteria for POEMS syndrome necessitates the presence of polyneuropathy and monoclonal gammopathy, in addition to at least one of the three major criteria (sclerotic bone lesions, Castleman's disease and elevated vascular endothelial growth factor levels) and one of the six minor criteria (organomegaly, extravascular volume overload, endocrinopathy, skin changes, papilloedema and thrombocytosis/polycythaemia).

The patient in this case study meets the criteria for a POEMS diagnosis. Other neurological manifestations of paraproteinaemias or plasma cell dyscrasias are: radiculopathy and spinal cord compression due to vertebral fractures or local extension of plasmacytoma (5\% cases of multiple myeloma), cranial and dural plasmacytoma, leptomeningeal myelomatosis ( $1 \%$ cases of multiple myeloma), cerebral vein thrombosis, stroke due to hyperviscosity and pachymeningitis. However, primary central nervous system amyloidosis is extremely rare. ${ }^{9}$

This patient developed heavy rectal bleeding following rectal biopsy, which is also a complication of amyloidosis. Such bleeding can arise as a result of many factors. This can be due to amyloidosis-related angiopathy arising from vascular fragility caused by vascular amyloid infiltration, impaired vasoconstriction following trauma caused by amyloid autonomic neuropathy, coagulation factor deficiency due to amyloid, liver impairment caused by marrow infiltration reducing synthesis, hyperfibrinolysis, platelet dysfunction and thrombocytopenia. ${ }^{10}$ Isolated acquired factor $X$ deficiency was found to be the most common coagulopathy in amyloidosis, which occurs via adsorption of factor $X$ to amyloid fibrils, leading to loss from 
the circulation. Deficiencies of coagulation factors V, II, VII and Von Willebrand factor are also reported."

The patient in this case study presented with severe hypoglycaemia, for which there may be many underlying reasons. In the case of nephropathy, renal impairment can reduce insulin excretion and thus predispose the patients to some degree of hypoglycaemia if they are receiving therapeutic insulin or sulphonylurea. This is unlikely to be true for the patient in this case study, as she has not developed significant renal impairment. However, she already has renal involvement, so she has the tendency to develop renal impairment in the future, which can predispose her to hypoglycaemia if she is on insulin or sulphonylureas.

The patient has a urine protein creatinine ratio of 2526 indicating significant proteinuria, but not meeting the definition of nephrotic syndrome, as protein excretion is less than 3,500 mg daily. The average urine protein excretion in cases of amyloid nephropathy is 4,000 mg daily. ${ }^{12}$ This patient has nephropathy with significant proteinuria in the absence of diabetic retinopathy, has lived with diabetes for a relatively short duration of time, and has upper normal serum creatinine levels. When considered together, these facts point towards amyloidosis being the cause of the patient's nephrotic syndrome, rather than diabetes mellitus. The prevalence of non-diabetic renal disease in patients with diabetes varies from $20-80 \%$ cases. ${ }^{13}$ The kidney is affected in $50-80 \%$ of amyloidosis cases. ${ }^{14}$ Renal failure is found to occur in $20-40 \%$ cases of multiple myeloma via various mechanisms. ${ }^{15}$ Excess light chains form tubular casts with Tamm-Horsfall proteins and cause obstruction of the distal convoluted tubule and the loop of Henle. Monoclonal light chains have a direct toxic effect on the proximal convoluted tubule, leading to reabsorption defects and resulting in acquired Fanconi syndrome, which in turn causes glycosuria, aminoaciduria, hypophosphataemia, and hypouricaemia. These mechanisms comprise a potential cause for hypoglycaemia in the patient in this case study. Lambda light chain, as identified in this patient, is responsible for $80 \%$ of renal amyloidosis cases in myeloma, and results in glomerulonephritis. In contrast, glomerulonephritis due to light chain deposition disease is mostly due to kappa light chains. Hypercalcaemia due to myeloma impairs the kidneys reabsorption ability, leading to hypovolaemia and renal failure. ${ }^{15}$ Renal involvement can be the presenting feature in $50 \%$ of cases of amyloidosis. Renal amyloidosis can present either in the form of glomerulonephritis (the most common), followed by tubular interstitial nephritis and renal vasculitis. Lambda-AL amyloidosis is associated with lower serum creatinine levels and higher proteinuria compared to kappa-AL amyloidosis, as was true in this case. ${ }^{16}$

Adrenal involvement in amyloidosis can also cause hypoglycaemia, hypotension and hyponatraemia, but was deemed unlikely in this case due to the patient's normal early morning cortisol levels, and her normal serum potassium levels. ${ }^{17}$ Monoclonal antibodies of multiple myeloma and other plasma cell dyscrasias have also rarely been found to have auto antibodies to indigenous insulin, resulting in hypoglycaemia. ${ }^{18}$

\section{Conclusion}

This patient has multi-organ involvement leading to autonomic instability, hypoglycaemia and bleeding tendency as a result of both multiple myeloma and the co-existence of amyloidosis, which is a known complication of myeloma. There should be a high index of suspicion for multiple myeloma and amyloidosis in patients who present with such multi-organ involvement. $\square$
Westermark P, Benson MD, Buxbaum JN, et al. A primer of amyloid nomenclature. Amyloid. 2007;14:179-83.

2. Hazenberg BP. Amyloidosis: a clinical overview. Rheum Dis Clin North Am. 2013;39:323-45

3. Skinner M, Sanchorawala V, Seldin DC, et al. High-dose melphalan and autologous stem-cell transplantation in patients with AL amyloidosis: An 8-year study. Ann Intern Med. 2004;140:85-93.

4. Kyle RA, Gertz MA. Primary systemic amyloidosis: clinical and laboratory features in 474 cases. Semin Hematol. 1995;32:45-9.

5. Rajkumar SV, Gertz MA, Kyle RA. Primary systemic amyloidosis with delayed progression to multiple myeloma. Cancer. 1998;82:1501-5.

6. Shin SC, Robinson-Papp J. Amyloid neuropathies. Mt Sinai J Med. 2012;79:733-48.

7. Nisar MU, Asad A, Waqas A, et al. Association of diabetic neuropathy with duration of type 2 diabetes and glycemic control Cureus Journal of Medical science. 2015;7:302.

8. Dalakas MC. Advances in the diagnosis, immunopathogenesis and therapies of IgM-anti-MAG antibody-mediated neuropathies. Ther Adv Neurol Disord. 2018;11:1756285617746640.

9. Velasco R, Bruna J. Neurologic complications of multiple myeloma and plasmacytoma. European Association of Neurooncology Magazine. 2012;2:71-77.

10. Sucker C, Hetzel GR, Grabensee B, et al. Amyloidosis and bleeding: pathophysiology, diagnosis, and therapy. Am J Kidney Dis. 2006;47:947-55.

11. Tripathi P, Pati HP, Tyagi S, et al. Amyloidosis presenting as severe bleeding diathesis. International Journal of Medicine and severe bleeding diathesis. Intern
Dental Sciences. 2017;6:1535-8.

12. Kuroda T, Tanabe N, Kobayashi D, et al. Significant association between renal function and amyloid-positive area in renal biopsy specimens in AL amyloidosis. BMC Nephrol. 2012;13:118.
13. Tone A Shikata $K$, Matsuda $M$, et al Clinical features of non-diabetic renal diseases in patients with type 2 diabetes. Diabetes Res Clin Pract. 2005;69:237-42.

14. Obici L, Perfetti V, Palladini G, et al. Clinical aspects of systemic amyloid diseases. Biochim Biophys Acta. 2005;1753:11-22.

15. Dimopoulos MA, Kastritis E, Rosinol L, et al. Pathogenesis and treatment of renal failure in multiple myeloma. Leukemia. 2008;22:1485-93.

16. Khalighi MA, Dean Wallace W, Palma-Diaz MF. Amyloid nephropathy. Clin Kidney J. 2014;7:97-106.

17. Piskinpasa SV, Akoglu H, Yenigun E et al. Adrenal insufficiency in a patient with end stage renal disease due to secondary amyloidosis. Turk Nephrol. 2013; 22:310-12.

18. Redmon B, Pyzdrowski KL, Elson MK, et al. Brief report: hypoglycemia due to a monoclonal insulin-binding antibody in multiple myeloma. N Eng/ I Med. 1992;326:994-8. 